\title{
Neurogenic diabetes insipidus presenting in a patient with subacute liver failure: a case report
}

\author{
Manu Shankar Hari ${ }^{1}$, Anthony K Parsons ${ }^{1}$, Andy K Burroughs ${ }^{2}$, Steve Shaw ${ }^{1}$, James O'Beirne ${ }^{2}$, Banwari Agarwal ${ }^{*}$
}

\begin{abstract}
Introduction: To the best of our knowledge, this is the first report in the literature of development of neurogenic diabetes insipidus in a patient with subacute liver failure.

Case presentation: A 25-year-old man presented with subacute liver failure. While awaiting a liver transplant, the patient developed cerebral edema, which resulted in neurogenic diabetes insipidus secondary to cerebral edema. The patient died before the liver transplantation could be carried out.

Conclusion: Neurogenic diabetes insipidus is well recognized in the neurosurgical population as a consequence of cerebral edema and increased intracranial pressure, both of which occur commonly in patients with subacute liver failure.
\end{abstract}

\section{Introduction}

Cerebral edema occurs in patients presenting with fulminant liver failure, resulting in increased intracranial pressure (ICP). The incidence and severity of cerebral edema increases as the onset of liver failure becomes rapid. It occurs in up to $80 \%$ of patients with acute and hyperacute fulminant liver failure but less frequently $(20 \%)$ in those with subacute fulminant liver failure. There is a significant association between the presence of cerebral edema and the development of central diabetes insipidus in patients with traumatic brain injury [1] and in postoperative neurosurgical patients. We present a case of neurogenic diabetes insipidus that developed during the course of subacute fulminant liver failure.

\section{Case presentation}

A 25-year-old Nigerian man was admitted to our hospital's intensive care unit (ICU), after emergency tracheal intubation and ventilation for worsening encephalopathy and a deteriorating Glasgow Coma Scale score. The working diagnosis was seronegative hepatitis leading to subacute fulminant liver failure and grade three hepatic encephalopathy.

\footnotetext{
* Correspondence: banwari.agarwal@royalfree.nhs.uk
${ }^{1}$ Intensive Care Unit, The Royal Free Hospital NHS Trust, Pond Street,

* Correspondence: banwari.agarwal@royalfree.nhs.uk
${ }^{1}$ Intensive Care Unit, The Royal Free Hospital NHS Trust, Pond Street, London, NW3 2QG, UK
}

(C) 2010 Hari et al; licensee BioMed Central Ltd. This is an Open Access article distributed under the terms of the Creative Commons

His admission to our hospital was preceded by an admission to a local hospital with a ten day history of painless jaundice and malaise, progressing to three days of nausea and vomiting. The patient had no history of encephalopathy or coagulopathy. Liver function tests on admission were abnormal (bilirubin, $381 \mu \mathrm{mol} / \mathrm{L}$, alanine transferase [ALT], 684 U/L), but an ultrasound scan showed a normal liver. Autoimmune and viral screen results were negative except for anti-smooth muscle antibody, which was 1 in 40 dilutions. He was sickle cell negative, and virology screening results were also negative. He had coagulopathy with an International Normalized Ratio (INR) above ten, which responded to vitamin $\mathrm{K}$ and fresh-frozen plasma (FFP). The patient had no history of any recent foreign travel, intravenous (IV) drug use, excessive alcohol intake or any family history of liver or autoimmune disease. The patient was discharged home five days after admission with plans for outpatient follow-up.

Six weeks after the initial presentation, the patient was readmitted to the same local hospital with continuing malaise, reduced appetite, vomiting, abdominal pain, worsening jaundice and confusion. The liver function tests on this occasion were bilirubin, 597; ALT, 475; alkaline phosphatase (ALP), 859; albumin, nine; and INR above nine. Results of blood films for malaria were negative. The patient was intubated and ventilated in the ICU before being transferred to our hospital on 
three weeks after this admission. He was found to have grade three hepatic encephalopathy. A repeat ultrasound scan of his abdomen revealed mild hepatosplenomegaly. A computed tomography (CT) scan of his head before transfer was normal, and CT scans of his thorax and abdomen showed a normal-looking liver, splenomegaly and coeliac lymphadenopathy.

On admission to our ICU, the patient was hemodynamically stable and was ventilated on pressure-controlled synchronized intermittent mandatory ventilation (PCSIMV) to achieve normocapnia $\left(\mathrm{PaCO}_{2}, 4.0-4.5\right.$ $\mathrm{kPa})$. His urea and creatinine levels at admission were $2.8 \mathrm{mmol} / \mathrm{L}$ and $112 \mu \mathrm{mol} / \mathrm{L}$, respectively. His INR was 4.4 after correction with FFP and vitamin K. Paracetamol levels were undetectable, and the serum lactate level was normal.

The patient was diagnosed with seronegative (virology screen negative) subacute (jaundice and encephalopathy $>$ eight weeks) fulminant liver failure, such that he was listed for urgent liver transplantation as the satisfied the King's College criteria for transplantation in acute liver failure unrelated to paracetamol overdose [2].

After the patient was listed for urgent liver transplant, a retrograde jugular bulb catheter was inserted into the patient to monitor his jugular venous oxygen saturation and to optimize cerebral perfusion management. His jugular venous saturation was $91 \%$, suggesting cerebral hyperemia. The management as per our ICU protocol included mild hypothermia $\left(35^{\circ} \mathrm{C}\right)$, deep IV sedation and mean arterial pressure of below $85 \mathrm{~mm} \mathrm{Hg}$. This reduced his $\mathrm{S}_{\mathrm{jv}} \mathrm{O}_{2}$ to $73 \%$, which was followed by a sustained increase of more than $85 \%$. The patient's serum ammonia level at this time was $170 \mu \mathrm{mol} / \mathrm{L}$ (reference range, $10-50 \mu \mathrm{mol} / \mathrm{L}$ ). In keeping with our standard practice for the management of acute liver failure, an extradural Camino ICP bolt was inserted to monitor his ICP. The opening ICP was $15 \mathrm{~mm} \mathrm{Hg}$, which settled at $89 \mathrm{~mm} \mathrm{Hg}$ with conservative management using a clear pressure waveform.

Over the next 24 hours, the patient became progressively polyuric, with a urine output ranging between 300 and $450 \mathrm{~mL} / \mathrm{h}$. He had not received mannitol or other diuretics during his admission to the ICU. His serum sodium level increased to $168 \mathrm{mmol} / \mathrm{L}$, and his urea and glucose levels were at $3 \mathrm{mmol} / \mathrm{L}$ and $3.6 \mathrm{~mol} / \mathrm{L}$, respectively. His paired measured plasma was $368 \mathrm{mOsmol} / \mathrm{L}$ and urine osmolalities were $175 \mathrm{mOsmol} / \mathrm{L}$. The polyuria was initially treated with water via nasogastric tube and with IV electrolyte-free solutions. A diagnosis of central diabetes insipidus was then made. Nephrogenic diabetes insipidus was excluded because of the absence of drugs known to cause it (the patient was being given piperacillin/tazobactam [Tazocin], fluconazole, pantoprazole and lactulose), normal blood levels of potassium and calcium ions, and the resolution of polyuria in response to desmopressin therapy.

Approximately 24 hours after the onset of neurogenic diabetes insipidus, the patient's pupils became progressively more dilated and unreactive to light. His ICP, however, remained stable at $15 \mathrm{~mm} \mathrm{Hg}$. After a bolus of mannitol $(0.5 \mathrm{~g} / \mathrm{kg})$, the patient was transferred to the radiology suite. A CT brain scan revealed a relatively tight supratentorial compartment with evidence of edema but with a minimal change in his infratentorial compartment and widely patent prepontine ambient cisterns. No acute bleed was identified (Figures 1 and 2).

A neurology consultation was sought because the patient now had neurogenic diabetes insipidus, fixed dilated pupils and supratentorial cerebral edema with maximal therapy, normal ICP, and no surgically treatable intracranial pathology on a background of subacute fulminant liver failure. The neurology opinion was that the patient had most likely experienced an irreversible transtentorial brain herniation despite an apparently normal ICP; thus, the likelihood of favorable neurologic recovery was minimal. A joint decision with the transplant team was then made to remove the patient from the transplant list. The patient died approximately 24 hours later.

Postmortem examination revealed "mild" cerebral edema with the presence of Alzheimer-type II astrocytes, suggesting hepatic encephalopathy. Histologic examination of the liver confirmed acute massive confluent hepatic necrosis with no evidence of chronic liver disease, possibly suggestive of an autoimmune etiology.

\section{Discussion}

Hepatic encephalopathy in patients with fulminant liver failure is characterized by changes in cerebral blood flow, cerebral edema and intracranial hypertension. Cerebral blood flow may increase in patients with fulminant hepatic failure because of gradual cerebral arteriolar vasodilatation and may lead to cerebral hyperemia and jugular venous oxygen saturations above $75 \%$ [3]. The prognosis for patients with such cerebral "luxury perfusion" and cerebral edema is very poor [4].

Cerebral edema in patients with fulminant liver failure is a characteristically cytotoxic edema resulting primarily from astrocyte swelling. This was demonstrated in our patient during the postmortem examination [5]. Increased ICP associated with cerebral edema is a common complication of fulminant liver failure. Clinical signs of cerebral edema and intracranial hypertension in patients with metabolic encephalopathy are neither specific nor sensitive. Features commonly seen are pupillary abnormalities, exaggerated reflexes and hemodynamic instability. A head CT scan frequently demonstrates cerebral edema in acute liver failure patients with 


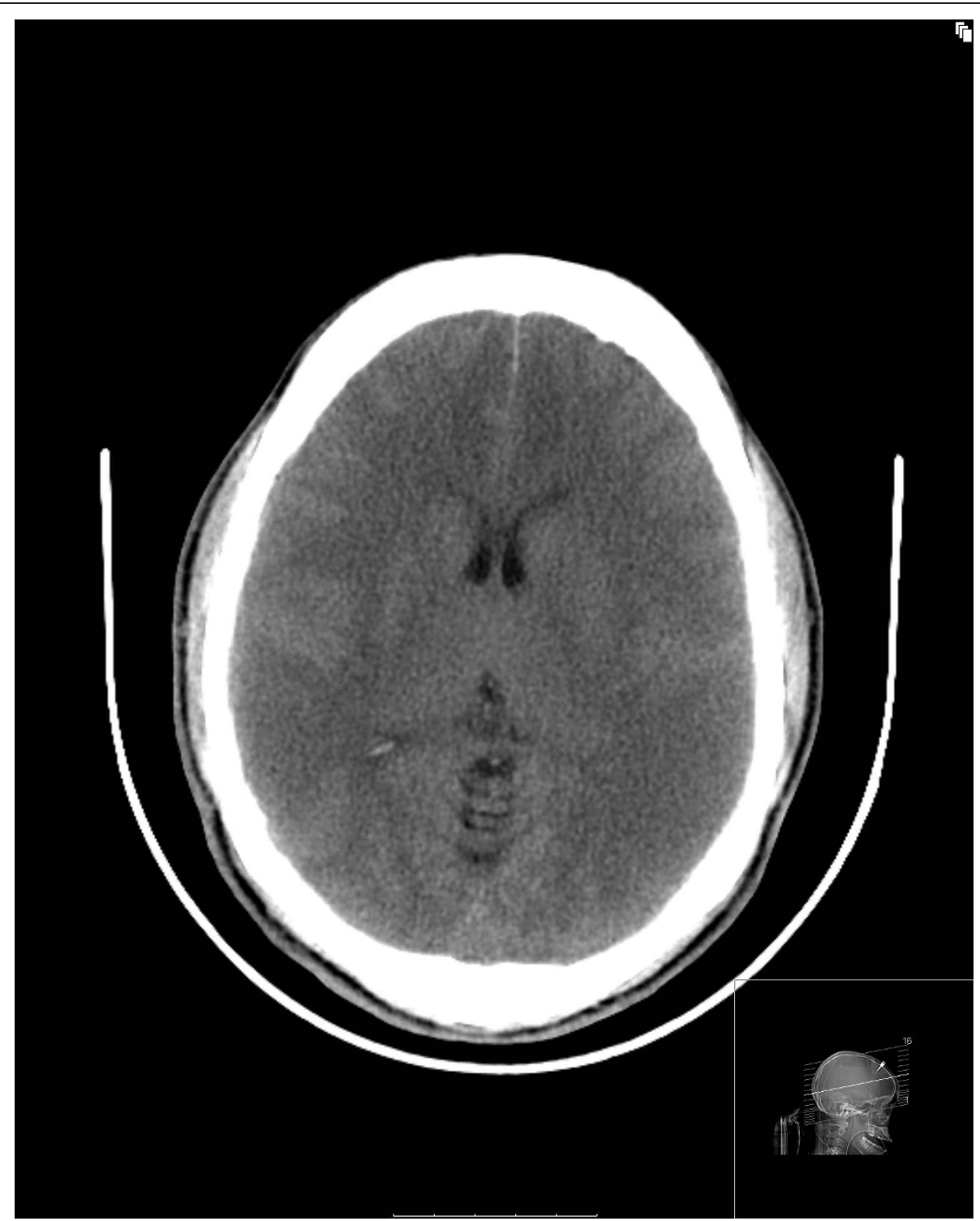

Figure 1 Computed tomography image showing significant edema predominantly in the supratentorial region.

advanced-stage hepatic encephalopathy [6] but is insensitive to intracranial hypertension [7]. In addition, clinical signs of intracranial hypertension, such as decerebrate posturing and sluggish pupillary responses, have been seen at ICPs of only 15 to $16 \mathrm{~mm} \mathrm{Hg}$ in patients with fulminant hepatic failure and cerebral edema [7].

Neurogenic diabetes insipidus is an uncommon condition. It is usually caused by lesions in the pituitary or supraoptic gland and paraventricular nuclei. It has also been identified in a variety of conditions. Neurogenic diabetes insipidus is defined as a decreased secretion of arginine vasopressin (AVP) leading to a high urine output of greater than $30 \mathrm{~mL} / \mathrm{kg}$ per 24 hours [4]. For polyuria to become clinically apparent, $80 \%$ of AVPsecreting neurons must be destroyed. The pathophysiology of posttraumatic neurogenic diabetes insipidus may involve increased ICP and cerebral edema around the hypothalamus or posterior pituitary gland, which explains the occurrence of diabetes insipidus in our patient [1].

\section{Conclusions}

To the best of our knowledge, there are no reports in the literature of neurogenic diabetes insipidus associated with cerebral edema from fulminant liver failure. Our patient had evidence of metabolic encephalopathy, cerebral hyperemia and cerebral edema. The cerebral edema 


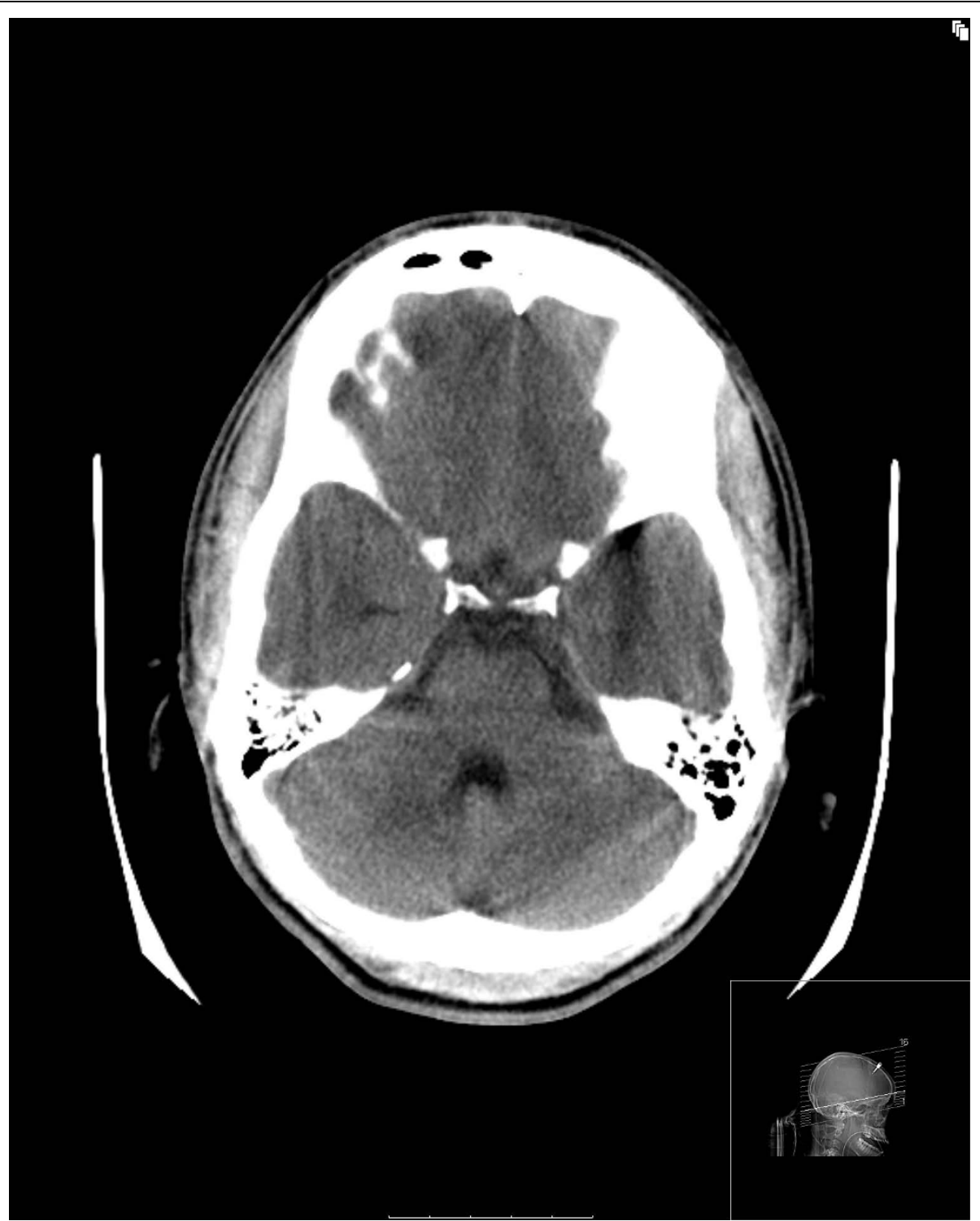

Figure 2 Computed tomography image showing relative sparing of the infratentorial compartment

had a relatively supratentorial distribution. The anatomical proximity of edema to the neurohypophysis most likely resulted in neurogenic diabetes insipidus.

\section{Consent}

Written informed consent could not be obtained despite all reasonable attempts as next of kin could not be contacted. Every effort has been made to protect the identity of the patient and there is no reason to think that the patient or their family would object to this publication.

\section{Abbreviations}

ALT: alanine transferase; ALP: alkaline phosphatase; AVP: arginine vasopressin; CT: computed tomography; FFP: fresh-frozen plasma; ICP: intracranial pressure; ICU: intensive care unit; INR: International Normalized Ratio; IV: intravenous; PCSIMV: pressure-controlled synchronized intermittent mandatory ventilation;

\section{Acknowledgements}

We thank the patient and all the members of the ICU team involved in his management.

\section{Author details}

${ }^{1}$ Intensive Care Unit, The Royal Free Hospital NHS Trust, Pond Street, London, NW3 2QG, UK. ${ }^{2}$ Liver Transplantation and Hepato-Biliary Medicine Unit, The Royal Free Hospital NHS Trust, Pond Street, London, NW3 2QG, UK.

\section{Authors' contributions}

MSH and AKP analyzed and interpreted patient data regarding the ICU management. $\mathrm{AKB}, \mathrm{SS}, \mathrm{JO}$ and $\mathrm{BA}$ were major contributors in writing the manuscript. All authors read and approved the final manuscript.

\section{Competing interests}

The authors declare that they have no competing interests. 


\section{References}

1. Agha A, Thornton E, O'Kelly P, Tormey W, Phillips W, Thompson CJ: Posterior pituitary dysfunction after traumatic brain injury. J Clin Endocrinol Metab 2004, 89:5987-5992.

2. Pauwels A, Mostefa-Kara N, Florent C, Levy VG: Emergency liver transplantation for acute liver failure. Evaluation of London and Clichy criteria. J Hepatol 1993, 17:124-127.

3. Larsen FS, Adel Hansen B, Pott F, Ejlersen E, Secher NH, Paulson OB, Knudsen GM: Dissociated cerebral vasoparalysis in acute liver failure. A hypothesis of gradual cerebral hyperaemia. J Hepatol 1996, 25:145-151.

4. Aggarwal S, Kramer D, Obrist W, Kang Y, Martin M, Policare R: Cerebral hemodynamic and metabolic changes in fulminant hepatic failure: a retrospective study. Hepatology 1994, 19:80-87.

5. Ranjan P, Mishra AM, Kale R, Saraswat VA, Gupta RK: Cytotoxic edema is responsible for raised intracranial pressure in fulminant hepatic failure: in vivo demonstration using diffusion-weighted MRI in human subjects. Metab Brain Dis 2005, 20:181-192.

6. Wijdicks EF, Plevak DJ, Ravel J, Wiesner RH: Clinical and radiologic features of cerebral edema in fulminant hepatic failure. Mayo Clin Proc 1995, 70:119-124.

7. Lidofsky SD, Bass NM, Prager MC: Intracranial pressure monitoring and liver transplantation for fulminant hepatic failure. Hepatology 1992, 16:1-7.

doi:10.1186/1752-1947-4-232

Cite this article as: Shankar Hari et al:: Neurogenic diabetes insipidus presenting in a patient with subacute liver failure: a case report. Journal of Medical Case Reports 2010 4:232.

\section{Submit your next manuscript to BioMed Central and take full advantage of:}

- Convenient online submission

- Thorough peer review

- No space constraints or color figure charges

- Immediate publication on acceptance

- Inclusion in PubMed, CAS, Scopus and Google Scholar

- Research which is freely available for redistribution

Submit your manuscript at www.biomedcentral.com/submit 